\title{
Phylogenetic Characteristics of Echinococcus granulosus Sensu Lato in Uzbekistan
}

\author{
Hye-Jin Kim, ${ }^{1,2}$, Tai-Soon Yong ${ }^{4}$, Myeong Heon Shin ${ }^{4}$, Kyu-Jae Lee ${ }^{5}$, Gab-Man Park ${ }^{6}$, Uktamjon Suvonkulov ${ }^{7}$, \\ Dmitriy Kovalenko ${ }^{7}$, Hak Sun $\mathrm{Yu}^{1,3, *}$ \\ 'Department of Parasitology and Tropical Medicine, Pusan National University, Yangsan 50612, Korea; 'Biological Resources Research Group, \\ Bioenvironmental Science \& Toxicology Division, Gyeongnam Branch Institute, Korea Institute of Toxicology (KIT), Jinju 52834, Korea; ${ }^{3}$ Research \\ Institute for Convergence of Biomedical Science and Technology, Pusan National University Yangsan Hospital, Yangsan 50612, Korea; ${ }^{4}$ Department \\ of Environmental Medical Biology and Institute of Tropical Medicine, Yonsei University College of Medicine, Seoul 03722, Korea; ${ }^{5}$ Department of \\ Environmental Medical Biology, Yonsei University Wonju College of Medicine, Wonju 26426, Korea; ${ }^{6}$ Department of Environmental Medical Biology, \\ Catholic Kwandong University College of Medicine, Gangneung 25601, Korea; ${ }^{7}$ saev Research Institute of Medical Parasitology, Ministry of Health, \\ Republic of Uzbekistan
}

\begin{abstract}
Echinococcosis occurs mainly in areas with heavy livestock farming, such as Central Asia, America, and Australia. Echinococcus granulosus sensu lato (s.l.) infection causes echinococcosis in intermediate hosts, such as sheep, cattle, goats, camels, and horses. Numerous cases of echinococcosis occur in Uzbekistan as stock farming is a primary industry. Epidemiological and genetic studies of $E$. granulosus s.l. are very important for mitigating its impact on public health and the economy; however, there are no such studies on E. granulosus s.l. in Uzbekistan. In the present study, to determine which genotypes exist and are transmitted, we isolated Echinococcus sp. from definitive hosts (one isolate each from jackal and dog) and intermediate hosts (52 isolates from humans and 6 isolates from sheep) in Uzbekistan and analyzed the isolates by sequencing 2 mitochondrial DNA components (cox1 and nad1). The results showed that all of isolates except one belonged to the $E$. granulosus sensu stricto (s.s.) G1 and G3 genotypes. Phylogenetic analysis based on cox 1 sequences showed that 42 isolates from humans, 6 isolates from sheep, and one isolate from jackal were the G1 genotype, whereas the remaining 8 isolates from human and the one isolate from dog were the G3 genotype. These results suggest that the G1 and G3 genotypes of $E$. granulosus s.s. are predominant in Uzbekistan, and both wild animals and domestic animals are important for maintaining their life cycle. Only one isolate from human sample was confirmed to be $E$. eqiinus (G4 genotype), which is known to be for the first time.
\end{abstract}

Key words: Echinococcus granulosus, Echinococcus equinus, genotype, Uzbekistan

Echinococcus granulosus sensu lato (s.l.) is a zoonotic parasite that infects a wide range of hosts, including sheep, dogs, cattle, and humans, and causes cystic echinococcosis (CE). To maintain their lifecycle, E. granulosus s.l. requires 2 mammalian hosts, the definitive host (dogs and other canids) and an intermediate host (sheep, cattle, camels, and goats) [1]. Echinococcosis is an important issue for farming industries due to its economic and public health impact [2]. Molecular taxonomic studies of E. granulosus s.l. have been conducted using mitochondrial DNA regions such as the cytochrome c oxidase sub-

\footnotetext{
- Received 18 November 2019, revised 15 February 2020, accepted 18 March 2020. *Corresponding author (hsyu@pusan.ac.kr) (C) 2020, Korean Society for Parasitology and Tropical Medicine This is an Open Access article distributed under the terms of the Creative Commons Attribution Non-Commercial License (https://creativecommons.org/licenses/by-nc/4.0) which permits unrestricted non-commercial use, distribution, and reproduction in any medium, provided the original work is properly cited.
}

unit 1 (cox1) and NADH dehydrogenase subunit 1 (nad1) genes, which have been used to define the genotypes G1-G10 [3-6]. However, the features of G9 remain unclear [7,8]. These genotypes are currently used as standards for molecular classification and epidemiology analyses [9]. In addition, it has been recently shown that G2 is no longer a valid genotype as well, but micro variant of G3 is available [10].

In Central Asia, most of the agricultural lands are semi-arid, but there are mountain grasslands; therefore, the farming industries in the countries of this region are concentrated on pasture-based livestock production [11]. This provides favorable conditions for the transmission of Echinococcus species from livestock reservoirs. Since the collapse of the Soviet Union in 1991, CE has emerged as a major zoonosis in the region, and the incidence of CE in humans has significantly increased [11]. In Kazakhstan, studies of the prevalence of CE suggested that 
the Echinococcus sp., infection rates are 30-50\% in sheep, 7\% in cattle, $5-10 \%$ in strait dogs (final host), and $>20 \%$ in shepherd dogs [12]. Most infected dogs have the G1 genotype, but the G6-G7 genotype has also been detected in dog feces. In Iran, genotypes G1-G3 are the dominant types responsible for most human and animal infections [13,14]; G6 is the second most prevalent genotype in the region, and it is increasingly being detected in human cases. In Uzbekistan, the prevalence of CE in sheep increased from $45 \%$ in 1990 to $62 \%$ in 2002 [11]. Although more than 8,000 people have been treated for cystic echinococcosis since 2002 in the 14 states of Uzbekistan [15], few, if any, epidemiological investigations have been conducted. Epidemiological investigations are key for establishing the epidemiologic relationships among circulating isolates. The information gathered should be considered in the development of local cystic echinococcosis control and prevention programs. Therefore, this information has significant epidemiological relevance because of the wide distribution of Echinococcus species.

The aim of this study was to investigate the dominant geno- types of E. granulosus s.l. isolates from various hosts in Uzbekistan. Genotypic and phylogenetic analyses of E. granulosus s.l. isolates obtained from human patients, a jackal, sheep, and a dog in Uzbekistan were conducted to determine their genetic relationships.

The fluid from 52 hydatid cysts (10 from lungs and 42 from livers) were collected from patients who underwent medical treatment at Samarqand Hospital in Uzbekistan in 2016-2017. Approval was obtained from the patients for the use of the hydatid cyst fluid for genetic analysis via ethic committee of ISAEV institute. The protoscoleces were obtained after centrifugation of the hydatid cyst fluid for $5 \mathrm{~min}$ at 2,000 $\times \mathrm{g}$ and were stored at $-20^{\circ} \mathrm{C}$ until use. Many adult worms were obtained from the small intestine of a jackal by hunting at a nearby hill in Samarqand, Uzbekistan at the beginning of 2016. Six hydatid cysts were collected from the livers of sheep that were infected with E. granulosus s.l. in Samarqand, Uzbekistan in the winter of 2017. Many adult worms were also collected from a dog in Samarqand, Uzbekistan over the same time period. Each 10

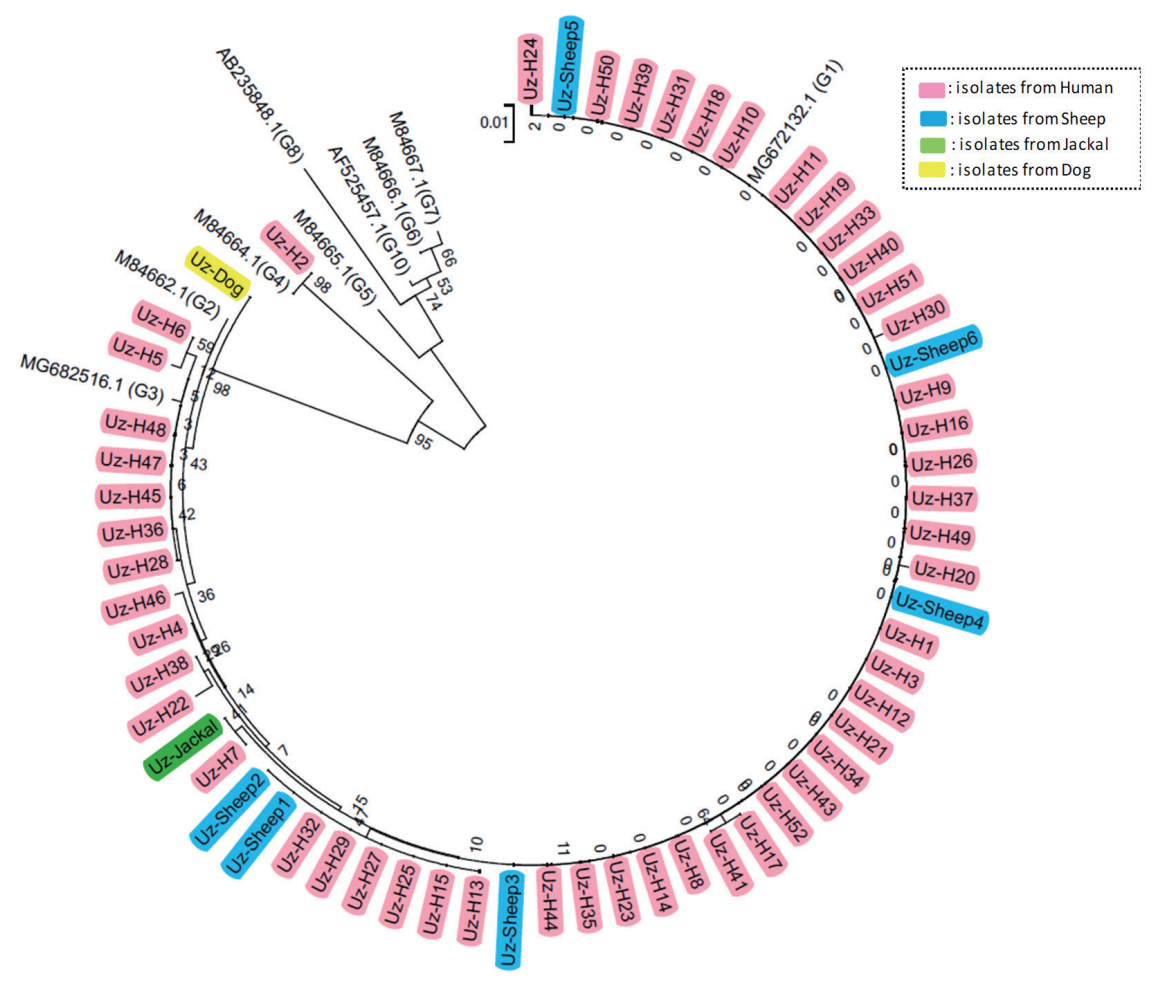

Fig. 1. Phylogenetic tree of Echinococcus granulosus isolates generated using cox 1 gene sequences. The genetic relationship among $E$. granulosus isolates was inferred based on a phylogenetic analysis using their cox1 sequences (GenBank assigned No. MK975892MK975951) along with 9 corresponding reference sequences. The phylogenetic tree was constructed by neighbor-joining method using MEGA version 6.0. The reference sequences are named by their GenBank accession number. The isolates are shown in different colors according to their host. 
adult worms from dog and jackal were stored $-20^{\circ} \mathrm{C}$ until use. Total genomic DNA was extracted from all of the collected samples with the QIAamp DNA Mini Kit (Qiagen, Hilden, Germany) according to the manufacturer's instructions. The extracted genomic DNA was stored at $-20^{\circ} \mathrm{C}$ until use. PCR was conducted using primer sets for cox 1 and nad 1 as described previously [16]. After purifying the PCR products with the Ex-

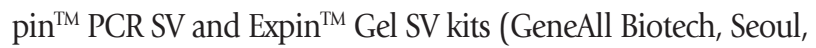
Korea), the cox1 and nad1 PCR products were analyzed by sequencing and phylogenetic analysis to confirm the genotype. The DNA sequencing was performed by Cosmo Genetech Co. (Cosmo Genetech, Seoul, Korea) using the $3730 \mathrm{xl}$ DNA Analyzer (Applied Biosystems, Foster City, California, USA). The reference sequences of the cox 1 and nad 1 genes of E. granulosus s.l. genotypes G1-G10 (except G9) were obtained from GenBank (https://www.ncbi.nlm.nih.gov/nucgss). Isolate and reference sequences were used in a multiple alignment, and phylogenetic distances were calculated using MEGA version 6.0. Phylogenetic trees were constructed using the neighbor-joining method in MEGA version 6.0. The all sequences were uploaded to GenBank and assigned GenBank number as cox1; MK 975892-MK975951, nad1; MN696570-MN696629).

Various protoscolex samples were obtained from final and intermediated hosts in Uzbekistan, including 52 samples from human patients, 1 sample from a jackal, 6 samples from sheep, and 1 sample from a dog. To identify the genotypes of the E. granulosus in the samples, we performed sequencing and phylogenetic analysis. Sequence analysis of the cox 1 and nad1 PCR products, including a multiple sequence alignment and phylogenetic analysis with reference sequences indicated that most of the isolates, except 1 sample (Uz-H2), had extremely high homology with genotype G1 and G3 E. granulosus s.s. reference sequences (Fig. 1, Supplementary Fig. S1). In contrast, Uz-H2 had extremely high homology with genotype G4 (E. equinus) reference sequences. However, the genetic distance between genotypes G1 and G3 is small, and thus, they could not be differentiated. To confirm the genotype (i.e., G1 or G3), we performed a detailed phylogenetic analysis using 5 G1 and 5

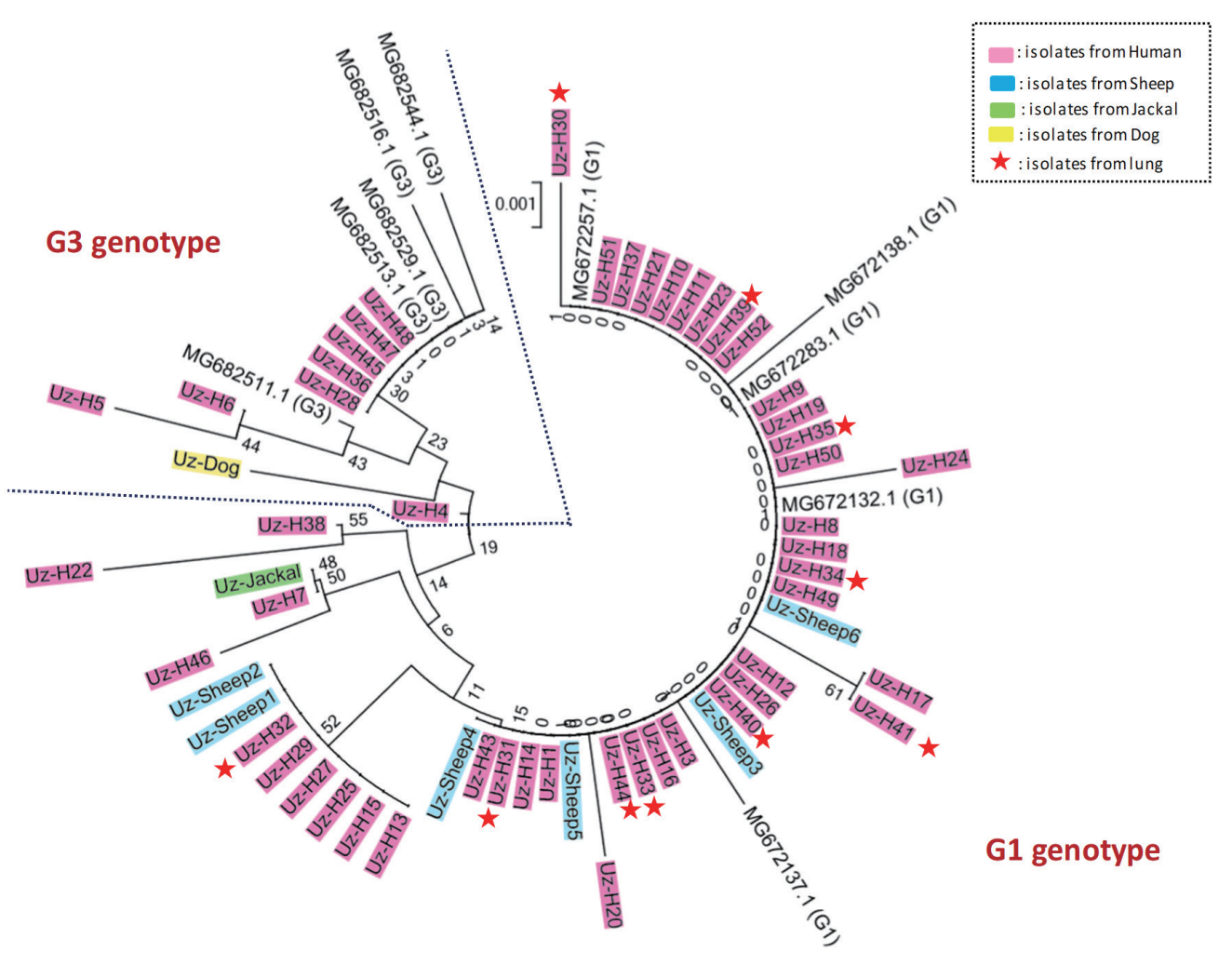

Fig. 2. Genetic relationships among G1 and G3 genotypes as determined by cox1 gene analysis. The genetic relationship was inferred based on phylogenetic analysis using the cox1 sequences of confirmed G1 and G3 genotypes. Except Uz-H2 (G4 genotype), all of samples were analyzed. The phylogenetic tree was constructed by neighbor-joining method using MEGA version 6.0. The isolates are shown in different colors according to their host. Red stars indicate human lung isolates. 
G3 isolates that were confirmed by near complete mitochondrial genome sequences in previous reports. As expected, 42 isolates from humans, all 6 isolates from sheep, and the 1 isolate from jackal were grouped with the confirmed G1 genotype sequences, while the remaining 8 isolates from humans and the 1 isolate from a dog were grouped with confirmed G3 genotype sequences (Fig. 2). To determine if the genotype of $E$. granulosus s.s. (i.e., G1 or G3) affects the organs that it can infect, we compared the genetic characteristics of the isolates according to the location of the cysts ( 10 from human lungs and 48 from the livers of humans and sheep). Interestingly, all isolates from the human lung grouped with confirmed G1 genotype strains.

Cases of CE in humans have been reported worldwide for more than 50 years. Until recently, the genus Echinococcus consisted of 9 valid species, including the livestock-friendly species [E. granulosus s.s. (G1 and G3), E. equinus (G4), E. ortleppi (G5), and E. canadensis (G6 and G7); species name is controversial ] and the wild animal-friendly species [E. canadensis (G8, G10), E. felidis, E. multilocularis, E. shiquicus, E. vogeli, and E. oligarthra] [7,8,17-19]. It is worth noting that genetic classification of isolates from both definitive and intermediate hosts can contribute to programs aimed to control this public health problem by determining how these organisms maintain their life cycle. Generally, there are many more genetic studies on livestock-friendly species because of their commonness. Previous studies revealed that E. granulosus s.s. G1 and G3 genotypes are the most common one infecting humans [20]. In fact, E. gramulosus s.s. G1 and G3 genotypes are the most common worldwide (72.9\%), followed by G6 and G7 genotypes (12.2\% and 9.6\%) [20]. In the Middle East, including in Iran and Kazakhstan, the G1 genotype is detected at a high frequency [12,21-23]. In this study, we firstly analyzed the genetic variation of E. granulosus s.l. isolates in Uzbekistan, and the results showed that G1 and G3 (E. granulosus s.s.) were the predominant genotypes among isolates from humans, sheep, and the definitive hosts (dog and jackal), and only 1 isolate was determined to be the G4 genotype (E. equinus).

The E. granulosus s.s. G1 and G3 genotypes are much more closely related to each other than to any other known genotype. Therefore, the sequences of cox 1 and nad1, which are most commonly used for typing, were too short to clearly identify our isolates as G1 and G3 [24,25]. Recently, successful differentiation of the G1 and G3 genotypes was achieved by using near-complete mitochondria genome sequences (11,443 bp) $[10,25]$. However, we successfully classified our isolates as G1 and G3 by using only the cox1 sequences (and not nad1) along with confirmed G1 and G3 genotype reference sequences. We used the cox1 gene region from the near complete mitochondria genomes, whose genotypes were confirmed in a previous study [25]. The complete mitochondrial genome sequences have more phylogenetic information than the short sequences used in this study. However, if confirmed G1 and G3 genotype sequences are used as reference sequences for E. granulosus s.s. genotyping, we can obtain results similar to those utilizing complete mitochondria genome sequences.

We confirmed the genotypes of the isolates from jackal, sheep, and dog as G1 and G3 and 1 isolate from human as G4 (Fig. 1, Supplementary Fig. S1). In this study, we obtained 3 notable results. First, we found that the transmissibility of the parasite in intermediated hosts might be determined by its genetic background. In this study, the 10 isolates from lungs were grouped with genotype G1. E. granulosus s.l. is known to form hydatid cysts in certain organs, especially in the liver and lungs [26]. Previous studies also showed an interesting result; $68 \%$ of the E. granulosus cysts in infected pigs were found in the lungs, whereas $73 \%$ of the cysts in cattle were found in the liver [27]. This markedly different organ distribution between the 2 species might be consistent with different genotypes circulating in different species [11]. Although we have no conclusive evidence to support the hypothesis that some genotypes are better able to transfer to the lung, in the present study, all isolates from the lung were grouped with the G1 genotype. To test this hypothesis, additional analyses using many more confirmed G1 and G3 sequences are needed. Second, infections by Echinococcus eggs from sheep and humans in the definitive hosts (dog and jackal) might be uncommon recently because the genetic distance between the isolates from jackal and dog and the isolates from sheep and human were great, although 2 isolates from human were very closely related to the jackal isolate. This result may determine which genotypes are the dominant cause of EC in Uzbekistan. The final notable finding is the first report of a human E. equinus infection. The biological differences in E. equinus when compared to E. granulosus s.l. have been confirmed [28]. This genotype was first detected as a parasite in a British horse, and it differed markedly in morphology compared to E. granulosus of sheep origin [29]. It seems to be a highly specific parasite of Equidae (horses, donkeys, and zebras), as all cases involving the G4 genotype, except one, were in the Equidae. Boufana et al. [30] first reported E. equinus infection in a primate (lemur), and in Jordan, although most dogs were infected with G1, a single sample was shown to be 
the most similar to E. equinus (G4) using RAPD-PCR [31]. These results suggest that $E$. equinus might have some potential for human infection in Middle Asia. We are currently conducting epidemiological investigations in Uzbekistan to determine if G4 genotype infections occur in horses or dogs in the region.

In conclusion, we confirmed that E. granulosus s.l.-infected jackal, sheep, and dogs may be associated with human infections. The results of this study will contribute to epidemiological surveys of E. granulosus s.l. in Uzbekistan, which will be helpful for improving public health and the economy.

\section{ACKNOWLEDGMENTS}

This work was supported by the Korea International Cooperation Agency (KOICA) under the project title "Capacity Building of Infectious and Parasitic Diseases Control in the Republic of Uzbekistan" in 2014 (no. P2014-00076-1).

\section{CONFLICT OF INTEREST}

The authors declare no conflict of interest related to this study.

\section{REFERENCES}

1. World Health Organization. Manual on Echinococcosis in $\mathrm{Hu}-$ mans and Animals: A Public Health Problem of Global Concern. Paros, France. WHO/OIE. 2001, pp 1-19.

2. Elmajdoub LO, Rahman WA, Fadzil M, Mohd SA. Studies on the protoscoleces and hooks of Echinococcus granulosus from Libya by scanning electron microscope. Acta Med Int 2014; 1: 74-81.

3. Bowles J, Blair D, McManus DP. Genetic variants within the genus Echinococcus identified by mitochondrial DNA sequencing. Mol Biochem Parasitol 1992; 54: 165-173.

4. Bowles J, McManus DP. NADH dehydrogenase 1 gene sequences compared for species and strains of the genus Echinococcus. Int J Parasitol 1993; 23: 969-972.

5. Bowles J, McManus DP. Genetic characterization of the Asian Taenia, a newly described taeniid cestode of humans. Am J Trop Med Hyg 1994; 50: 33-44.

6. Gasser RB, Zhu X, McManus DP. NADH dehydrogenase subunit 1 and cytochrome c oxidase subunit I sequences compared for members of the genus Taenia (Cestoda). Int J Parasitol 1999; 29: 1965-1970.

7. Knapp J, Gottstein B, Saarma U, Millon L. Taxonomy, phylogeny and molecular epidemiology of Echinococcus multilocularis: from fundamental knowledge to health ecology. Vet Parasitol 2015; 213: 85-91.
8. Nakao M, Lavikainen A, Yanagida T, Ito A. Phylogenetic systematics of the genus Echinococcus (Cestoda: Taeniidae). Int J Parasitol 2013; 43: 1017-1029.

9. McManus DP, Thompson RC. Molecular epidemiology of cystic echinococcosis. Parasitology 2003; 127 (suppl): 37-51.

10. Kinkar L, Laurimäe T, Sharbatkhori M, Mirhendi H, Kia EB, Ponce-Gordo F, Andresiuk V, Simsek S, Lavikainen A, Irshadullah M, Umhang G, Oudni-M'rad M, Acosta-Jamett G, Rehbein S, Saarma U. New mitogenome and nuclear evidence on the phylogeny and taxonomy of the highly zoonotic tapeworm Echinococcus granulosus sensu stricto. Infect Genet Evol 2017; 52: 52-58.

11. Deplazes P, Rinaldi L, Alvarez Rojas CA, Torgerson PR, Harandi MF, Romig T, Antolova D, Schurer JM, Lahmar S, Cringoli G, Magambo J, Thompson RC, Jenkins EJ. Global distribution of alveolar and cystic echinococcosis. Adv Parasitol 2017; 95: 315493.

12. Abdybekova A, Sultanov A, Karatayev B, Zhumabayeva A, Shapiyeva Z, Yeshmuratov T, Toksanbayev D, Shalkeev R, Torgerson PR. Epidemiology of echinococcosis in Kazakhstan: an update. J Helminthol 2015; 89: 647-650.

13. Harandi MF, Hobbs RP, Adams PJ, Mobedi I, Morgan-Ryan UM, Thompson RC. Molecular and morphological characterization of Echinococcus granulosus of human and animal origin in Iran. Parasitology 2002; 125: 367-373.

14. Sharbatkhori M, Fasihi Harandi M, Mirhendi H, Hajialilo E, Kia EB. Sequence analysis of cox1 and nad1 genes in Echinococcus granulosus G3 genotype in camels (Camelus dromedarius) from central Iran. Parasitol Res 2011; 108: 521-527.

15. Hong ST, Jin Y, Anvarov K, Khadjibaev A, Hong S, Ahmedov Y, Otaboev U. Infection status of hydatid cysts in humans and sheep in Uzbekistan. Korean J Parasitol 2013; 51: 383-385.

16. Kim HJ, Yong TS, Shin MH, Lee KJ, Park GM, Suvonkulov U, Kovalenko D, Yu HS. Practical Algorisms for PCR-RFLP-Based Genotyping of Echinococcus granulosus sensu lato. Korean J Parasitol 2017; 55: 679-684.

17. Thompson RC, McManus DP. Towards a taxonomic revision of the genus Echinococcus. Trends Parasitol 2002; 18: 452-457.

18. Thompson RC, Lymbery AJ, Constantine CC. Variation in Echinococcus: towards a taxonomic revision of the genus. Adv Parasitol 1995; 35: 145-176.

19. Laurimae T, Kinkar L, Moks E, Romig T, Omer RA, Casulli A, Umhang G, Bagrade G, Irshadullah M, Sharbatkhori M, Mirhendi H, Ponce-Gordo F, Soriano SV, Varcasia A, Rostami-Nejad M, Andresiuk V, Saarma U. Molecular phylogeny based on six nuclear genes suggests that Echinococcus granulosus sensu lato genotypes G6/G7 and G8/G10 can be regarded as two distinct species. Parasitology 2018; 145: 1929-1937.

20. Cucher MA, Macchiaroli N, Baldi G, Camicia F, Prada L, Maldonado L, Avila HG, Fox A, Gutierrez A, Negro P, Lopez R, Jensen O, Rosenzvit M, Kamenetzky L. Cystic echinococcosis in South America: systematic review of species and genotypes of Echinococcus granulosus sensu lato in humans and natural domestic hosts. Trop Med Int Health 2016; 21: 166-175. 
21. Thompson RCA, Deplazes P, Lymbery AJ. Advances in Parasitology. Echinococcus and Echinococcosis, Part A. London, UK. Zoe Kruze. 2017, pp 1-525.

22. Ito A, Budke CM. The echinococcoses in Asia: the present situation. Acta Trop 2017; 176: 11-21.

23. Sharma M, Sehgal R, Fomda BA, Malhotra A, Malla N. Molecular characterization of Echinococcus granulosus cysts in north Indian patients: identification of G1, G3, G5 and G6 genotypes. PLoS Negl Trop Dis 2013; 7: e2262.

24. Casulli A, Interisano M, Sreter T, Chitimia L, Kirkova Z, La Rosa G, Pozio E. Genetic variability of Echinococcus granulosus sensu stricto in Europe inferred by mitochondrial DNA sequences. Infect Genet Evol 2012; 12: 377-383.

25. Kinkar L, Laurimäe T, Acosta-Jamett G, Andresiuk V, Balkaya I, Casulli A, Gasser RB, van der Giessen J, González LM, Haag KL, Zait H, Irshadullah M, Jabbar A, Jenkins DJ, Kia EB, Manfredi MT, Mirhendi H, M'rad S, Rostami-Nejad M, Oudni-M'rad M, Pierangeli NB, Ponce-Gordo F, Rehbein S, Sharbatkhori M, Simsek S, Soriano SV, Sprong H, Šnábel V, Umhang G, Varcasia A, Saarma U. Global phylogeography and genetic diversity of the zoonotic tapeworm Echinococcus granulosus sensu stricto genotype G1. Int J Parasitol 2018; 48: 729-742.

26. Moro P, Schantz PM. Echinococcosis: a review. Int J Infect Dis.
2009; 13: 125-133.

27. Suleimanova K, Shinkina E. Questions about echinococcosis in cattle. In: Materials of an International Scientific Practical Conference in Celebration of 85 years of the Ural Government Academy of Veterinary Medicine and 100th Anniversary of the Birth of Professor Vasiliniya Grigoryevicha Martinova. Tronitsk, 2015, pp 70-73.

28. Kumaratilake LM, Thompson RC, Eckert J. Echinococcus granulosus of equine origin from different countries possess uniform morphological characteristics. Int J Parasitol 1986; 16: 529-540.

29. Sweatman GK, Williams RJ. Comparative studies on the biology and morphology of Echinococcus granulosus from domestic livestock, moose and reindeer. Parasitology 1963; 53: 339-390.

30. Boufana B, Stidworthy MF, Bell S, Chantrey J, Masters N, Unwin S, Wood R, Lawrence RP, Potter A, McGarry J, Redrobe S, Killick R, Foster AP, Mitchell S, Greenwood AG, Sako Y, Nakao M, Ito A, Wyatt K, Lord B, Craig PS. Echinococcus and Taenia spp. from captive mammals in the United Kingdom. Vet Parasitol 2012; 190: 95-103.

31. Al-Qaoud KM, Abdel-Hafez SK, Craig PS. Canine echinococcosis in northern Jordan: increased prevalence and dominance of sheep/dog strain. Parasitol Res 2003; 90: 187-191. 